\title{
Celecoxib reduces risk of ulcer bleeding
}

Concomitant use of aspirin augments the risk of upper gastrointestinal bleeding associated with NSAIDs, yet some patients require both treatments. A study assessing the gastrointestinal safety of NSAIDs in aspirin users has found that co-therapy with celecoxib plus a proton-pump inhibitor (PPI) is superior to the combination of naproxen and a PPI for the prevention of recurrent upper gastrointestinal bleeding in patients at high risk of both cardiovascular and gastrointestinal events who require anti-inflammatory analgesics. "Contrary to present guidelines, we found that naproxen cannot be recommended to these high-risk patients despite its perceived cardiovascular benefit," reports lead author Francis Chan.

The current guidelines for NSAID selection in these patients are conflicting, and either suggest avoiding all NSAIDs or recommend naproxen as the preferred NSAID, or do not offer any advice. However, Chan and colleagues point out

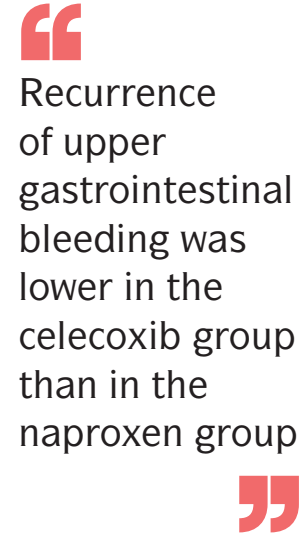

that these guidelines are not evidence-based, and that large-scale, industry-sponsored randomized trials have not been designed to address the choice of NSAID in this highrisk patient group. The industryindependent CONCERN study was undertaken to tackle this unmet need. "Unlike all other published trials that largely focused on averageto-moderate risk patients, we selectively recruited patients at high [gastrointestinal] risk (with a history of ulcer bleeding), requiring aspirin and NSAID [s] for cardiovascular and anti-inflammatory therapies, knowing that the choice of NSAID will be the most important aspect of the regime," explains Chan.

The single-centre study randomly allocated 514 patients with arthritis and cardiothrombotic diseases and a history of upper gastrointestinal bleeding to receive either the selective cyclooxygenase- 2 inhibitor celecoxib $100 \mathrm{mg}$ twice daily or the nonselective NSAID naproxen $500 \mathrm{mg}$ twice daily for 18 months. All patients also received aspirin $80 \mathrm{mg}$ daily and the PPI esomeprazole $20 \mathrm{mg}$ daily. Recurrence of upper gastrointestinal bleeding was lower in the celecoxib group than in the naproxen group (5.6\% versus $12.3 \%)$. The rate of adverse events leading to discontinuation of treatment was similar in both groups ( $8 \%$ versus $7 \%$ ), as was the incidence of cardiovascular events (4.4\% versus $5.5 \%$ ).

The authors note that co-therapy with celecoxib and a PPI reduces, but does not eliminate, the risk of recurrent bleeding in patients with a history of ulcer bleeding who require concomitant aspirin and NSAIDs. "[W]e believe our findings provide important novel data for various guideline committees towards revising their recommendations to improve our care for these high-risk patients," says Chan.

Sarah Onuora

ORIGINAL ARTICLE Chan, F. K. L. et al.

Gastrointestinal safety of celecoxib versus naproxen in patients with cardiothrombotic diseases and arthritis after upper gastrointestinal bleeding (CONCERN): an industry-independent, double-blind, double-dummy, randomised trial. Lancet http://doi.org/10.1016/S01406736(17)30981-9 (2017)

FURTHER READING Nurmohamed, M. T. et al.

Cardiovascular safety of celecoxib, naproxen and ibuprofen. Nat. Rev, Rheumatol. 13, 136-138 (2017) 